
AND CONTROLLED NUCLEAR FUSION RESEARCH

Seville, Spain, 26 September - 1 October 1994

IAEA-CN-60/ D-P-1-12

\title{
DITHERING TRANSITIONS IN RESISTIVE PRESSURE-GRADIENT-DRIVEN TURBULENCE
}

\author{
L. Garcia \\ Universidad Carlos III, Madrid, Spain \\ B.A. Carreras and V.E. Lynch \\ Oak Ridge National Laboratory, Oak Ridge, USA \\ P.H. Diamond* \\ University of California, San Diego, USA
}

\section{DISCLAIMER}

\begin{abstract}
This report was prepared as an account of work sponsored by an agency of the United States Government. Neither the United States Government nor any agency thereof, nor any of their employees, makes any warranty, express or implied, or assumes any legal liability or responsibility for the accuracy, completeness, or usefulness of any information, apparatus, product, or process disclosed, or represents that its use would not infringe privately owned rights. Reference herein to any specific commercial product, process, or service by trade name, trademark, manufacturer, or otherwise does not necessarily constitute or imply its endorsement, recommendation, or favoring by the United States Government or any agency thereof. The views and opinions of authors expressed herein do not necessarily state or reflect those of the United States Government or any agency thereof.
\end{abstract}

* Also General Atomics, San Diego, USA.

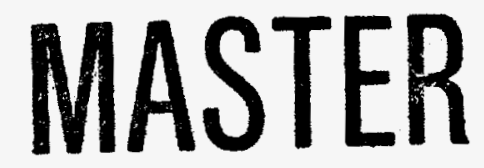

This is a preprint of a paper intended for presentation at a scientific meeting. Becaust of the provisional nature of its content and since changes of substance or detail may have to be made before publication. the preprint is made available on the understanding that it will not be cited in the literature or in any way be reproduced in its present form. The views expressed and the statements made remain the responsibility of the named author (s); the views do not necessarily reflect those of the government of the designating Member State(s) or of the designating organization(s). In particular, neither the (AEA nor any other arganization or body sponsoring this meeting can be held responsible for any material reproductd in this preprint. 


\section{DISCLAIMER}

Portions of this document may be illegible in electronic image products. Images are produced from the best available original document. 


\title{
DITHERING TRANSITIONS IN RESISTIVE PRESSURE-GRADIENT-DRIVEN TURBULENCE
}

\begin{abstract}
A self-consistent model of the $\mathrm{L}$ to $\mathrm{H}$ transition has been derived from coupled nonlinear envelope equations for the fluctuation level, and radial electric field shear, $E_{r}^{\prime}$. This model is based on general properties of the coupling between turbulence and averaged sheared flows. To extract these generic properties several turbulence models have been investigated. Here, some of the results obtained with the resistive pressure-gradient-driven turbulence are presented with special emphasis on the structure of the Reynolds stress and the existence of limit cycle solutions.
\end{abstract}

\section{INTRODUCTION}

Recently, a phase transition model [1] has been proposed to explain the transition from the low confinement mode (L-mode) to the high confinement mode (H-mode) [2] in toroidal devices. The model describes the simultaneous evolution of the fluctuation spectrum and the mean flow shear. In this model, the $\mathrm{L}$ to $\mathrm{H}$ transition appears as a second-order phase transition for which the order parameter is the radial electric field shear, $E_{r}^{\prime}$. This model is based on general properties of the coupling between turbulence and averaged sheared flows. To extract these generic properties several turbulence models have been investigated in detail. In particular, the parallel ion flow gradient driven instability that was discussed in reference 3 and the resistive pressure-gradient-driven turbulence. Some of the results obtained with the latter are presented in this paper.

Previous results [4] of resistive pressure-gradient-driven turbulence coupled with averaged shear flow evolution shown that self-consistent sheared flow $\left(V_{\theta}^{\prime} \neq 0\right.$ and $V_{\theta}^{\prime \prime}=0$ at the resonance surface) does not suppress turbulence in the nonlinear regime. However, curvature flows ( $V_{\theta}^{\prime}=0$ and $V_{\theta}^{\prime \prime} \neq 0$ at the resonance surface) are effective in turbulence suppression. These results were obtained with a fixed pressure gradient leading to large fluctuation levels $\left(\bar{n} / n_{0} \geq 0.1\right)$. Fluctuation suppression by an externally applied sheared flow [5] requires sheared flow levels an order of magnitude larger than what was expected from the sheared flow suppression theory of Biglari et al. [6]. In this paper, a different regime with low fluctuation levels $\left(\tilde{n} / n_{0} \leq 0.03\right)$ is investigated. This regime has been achieved by letting the averaged pressure gradient to evolve in time. In this regime, there are second order phase transitions of the type described by the model in reference 1 . Close to marginal stability, the Reynolds stress can be calculated. It consists of the so called $\alpha$ term as in the case of AKA instabilities [7] and an amplitude dependent turbulent viscosity term. At high fluctuation level, these two terms cancel each other. This cancellation explains the lack of second order transitions in high fluctuation level regime. 
The detailed dependence of the Reynolds stress and the shear suppression terms on the fluctuation amplitude are critical in explaining a dithering behavior of the transition for very low (very high) viscosity (power).

\section{RESISTIVE PRESSURE-GRADIENT-DRIVEN TURBULENCE EQUATIONS}

From the renormalized set of equations [8] and by averaging over the radius, assuming Gaussian dependence for the eigenfunctions, a two-equation system can be derived for the evolution of the fluctuation envelope. To these equation, we must add the poloidal shear flow and averaged presure evolution equations. A detailed derivation of these equations will be given elsewhere. The four equations are

$$
\begin{aligned}
& \frac{\partial \mathrm{E}}{\partial t}=2 \frac{W^{2}}{W_{0}^{2}} P-\left[\frac{W^{2}}{W_{0}^{2}}+3 \bar{\mu}+1.1 \lambda \mathrm{E}+\frac{\Omega_{s}^{2}}{2(2 \bar{D}+\mathrm{E})}\right] \mathrm{E} \\
& \frac{\partial P}{\partial t}=\left(1+\frac{N-1}{\sqrt{2}}\right) \mathrm{E}-(\bar{D}+0.54 \mathrm{E}) P \\
& \frac{\partial \Omega_{s}}{\partial t}=\left(\frac{1}{16} \frac{W^{2}}{W_{0}^{2}} g N-\frac{1}{6(\bar{\mu}+\lambda E / 3)}\right) \Omega_{s} \mathrm{E}^{2}-\frac{\hat{\mu}}{\gamma_{0}} \Omega_{s} \\
& \frac{\partial N}{\partial t}=\bar{Q}-4 \bar{D} N-\frac{1}{2 \bar{D}+\mathrm{E}} N \mathrm{E}^{2}
\end{aligned}
$$

where, $E=k_{\theta}|\tilde{\Phi}| /\left(\gamma_{0} W\right)$ and $P \equiv|\tilde{p}| /\left(-W p_{e q}^{\prime}\right)$ are the normalized velocity stream function and pressure fluctuation envelopes, $\Omega_{s} \equiv k_{\theta}\left\langle V_{\theta}\right\rangle^{\prime} W / \gamma_{0}$ is the normalized shearing rate [6], and $N \equiv\langle p\rangle^{\prime} / p_{e q}^{\prime}$ is the normalized averaged pressure gradient. Here, $\gamma_{0}$ the linear instability growth rate in the absence of collisional dissipation and sheared flow effects, $W$ and $W_{0}$ are the mode width with and without dissipation respectively, $k_{\theta}$ the poloidal component of the wavenumber vector, $p_{e q}^{\prime}$ is the equilibrium pressure gradient, and $\lambda \equiv k_{\theta} /\left\{k_{\theta}^{2}\right\}^{1 / 2}$. Here, the curly brackets, \{\} , indicate spectral average and the angular brackets, \langle\rangle , spatial average over the poloidal and toroidal angles. The normalized collisional dissipation coefficients are $\bar{\mu} \equiv \mu_{0} /\left(2 \gamma_{0} W^{2}\right)$ and $\bar{D} \equiv D_{0} /\left(2 \gamma_{0} W^{2}\right)$. In Eq. (3), $\hat{\mu}$ is the flow damping coefficient due to magnetic pumping and the prime indicate radial derivative. The function $g$ is $g \equiv[3 \bar{\mu}+\bar{D}+0.5(3 \lambda+1) \mathrm{E}] /\left[(\bar{\mu}+\lambda \mathrm{E} / 3)^{2}(\bar{D}+0.5 \mathrm{E})^{2}\right]$

These four equations, Eqs. (1)-(4), are the basic model equations. The phase transition model [1] equations are a simplified version of this model. The fixed points of this system of equations are essentially the same as for the equations in reference 1 . In this case, it is not possible to obtain compact analytical expressions. There are two types of stable fixed points, a fixed point with $\Omega_{\mathrm{s}}=0$, the L-mode type solution, and one with $\Omega_{\mathrm{s}} \neq 0$, the H-mode solution. The first fixed point is independent of $\hat{\mu}$ but the second depends on $\hat{\mu}$. The fluctuation amplitude $\mathrm{E}$ is a continuous function of $\hat{\mu}$ with non-continuous derivative at the critical point. 
This behavior is characteristic of a second order phase transition. The critical point is

$$
\hat{\mu}_{c r i t}=\gamma_{0}\left(\frac{1}{16} \frac{W^{2}}{W_{0}^{2}} g\left(\mathrm{E}_{L}\right) N_{L}-\frac{1}{6\left(\bar{\mu}+\lambda E_{L} / 3\right)}\right) \mathrm{E}_{L}^{2},
$$

where the subindex $L$ indicates that the $\mathrm{L}$-mode value for $\mathrm{E}$ and $N$ should be used in evaluating Eq. (5).

Numerical calculations using the full system of equations in reference 8 with coupled evolution of the averaged poloidal flow and averaged pressure show same characteristic behavior. In Fig. 1, we show the level of fluctuations $E$ at saturation as a function of $\hat{\mu}$ for the same equilibrium parameters as in Ref. 7 , with $\mu_{0}=0.2 a^{2} / \tau_{R}, D_{0}=0.05 a^{2} / \tau_{R}$, and $\beta_{0} / 2 \varepsilon^{2}=$ 0.0075 . The critical transition point is $\hat{\mu}_{c r i t}=2.0 / \tau_{R}$.

In deriving Eq. (3), we found that the Reynolds stress has two terms. One is proportional to the poloidal flow velocity and the second one proportional to its second derivative

$$
-\left.\frac{1}{r^{2}} \frac{\partial}{\partial r}\left(r^{2}\left\langle\tilde{V} \tilde{V}_{\theta}\right\rangle\right)\right|_{x=0}=\left.\langle\tilde{V} r \tilde{U}\rangle\right|_{x=0}=\alpha\left\langle V_{\theta}\right\rangle+\beta \frac{d^{2}\left\langle V_{\theta}\right\rangle}{d r^{2}}
$$

Here, $\alpha$ and $\beta$ are positive coefficients. The $\alpha$-term leads to a dynamo instability and the generation of flow. The $\beta$-term is an effective turbulent viscosity that damps poloidal flow. When an external sheared flow, $V_{\theta}=x V^{\prime}$, is applied, the second term is zero and sheared flow is generated. However, since the generated flow has no longer the linear $x$ dependence, the $\beta$ term becomes increasingly important and it cannot be neglected in the self-consistent flow evolution. For large fluctuations, E > 1, the two terms in Eq. (6) cancel each other and there is no flow generation. In this regime the resistive interchange instability remains always in the Lmode state, as it was found in reference 4 . For lower values of $E$, the cancellation is only partial. In general, the $\alpha$-term dominates and there is a potential increase in the sheared flow. The balance of the Reynolds stress with the velocity damping term gives the critical transition point. However, since both the $\alpha$ - and $\beta$-terms are large and of similar magnitude it is difficult to make an accurate prediction of the critical point based on this approximate model.

In calculating the correlation between the fluctuating radial velocity and the fluctuating vorticity, the $\alpha$-term comes from the instability drive in the fluctuation evolution equation while the $\beta$-term is due to the Kelvin-Helmholtz term. The association of the $\beta$-term with the KelvinHelmholtz term allows us to test the structure of the Reynolds stress. To do so, we calculate the critical transition point with and without the Kelvin-Helmholtz using the full equations. In the first case, the transition is given by the two terms in Eq. (6) while in the second case is only given by the $\alpha$-term. The numerical results for $\alpha$ and $\alpha-\beta / W_{v}^{2}$ calculated in this way are given in Fig. 2. Here, $W_{v}$ is the characteristic width of the velocity profile. 


\section{DITHERING SOLUTIONS}

For H-mode type solutions and when $\hat{\mu}$ decreases, one reaches a new regime characterized by the oscillatory behavior of the solutions. This happens for both the full equations solution (Fig. 3), and for the model in Section 2. By careful analysis of the model, it is possible to identify the oscillatory behavior with the nonlinear modification of the shearing term in the $\Phi$ fluctuation equation. We can understand that by using a further simplified model.

$$
\begin{aligned}
& \frac{\partial \mathrm{E}}{\partial t}=\left[1-\mathrm{E}-\frac{\Omega_{s}^{2}}{\delta+\mathrm{E}}\right] \mathrm{E} \\
& \frac{\partial \Omega_{s}}{\partial t}=a \Omega_{s} \mathrm{E}-b \Omega_{s}
\end{aligned}
$$

This model is a modification of the one given in reference 1 by adding the nonlinear weakening of the shearing effect in Eq. (7). This system of equations has the usual three fixed points: 1) $\mathrm{E}=\Omega_{\mathrm{s}}=0$ the trivial equilibrium solution, 2) $\Omega_{\mathrm{s}}=0$ and $\mathrm{E}=1$, the L-mode solution, and 3) $\mathrm{E}=b / a$ and $\Omega_{s}^{2}=(\delta+b / a)(1-b / a)$, the H-mode solutions. The basic properties of the first two fixed points are discussed in reference 1 . For the $\mathrm{H}$-mode fixed point, this system of equations verifies the conditions of the Kolmogorov theorem. Therefore, the H-mode solution is either a stable fixed point or a stable limit cycle. Linearizing Eqs. (7) and (8), we test the local stability properties of the H-mode fixed point. For $\delta>1-2 b / a$, the $\mathrm{H}$-mode fixed point is locally stable. Therefore, when $\delta<1-2 b / a$, the stable solution is a limit cycle. The numerical solution of Eqs. (7) and (8) confirms these results. The numerical calculations clearly show the existence of such solutions and the bifurcation threshold, $\hat{\mu}_{l c}$, from stable fixed point to a limit cycle is compatible with the relationship derived with the two equation model $\hat{\mu}_{l c}=\hat{\mu}_{c r i r}\left(1-D_{0} / \gamma_{0} W^{2}\right) / 2$. To reach this threshold, a growth rate (power) more than a factor of two larger than its critical value at the $\mathrm{L}$ to $\mathrm{H}$ transition is needed. Close to the bifurcation point to a limit cycle, the oscillation frequency can be estimate by a two scale expansion of the Eqs. (7) and (8). This gives:

$$
\frac{\omega^{2}}{\gamma_{0}^{2}}=2 b\left(1-\frac{b}{a}\right)+\frac{b}{a} \frac{1-\delta-2 b / a}{\delta+b / a}
$$

Near the bifurcation point, $\omega \approx \sqrt{2 \gamma_{0} \hat{\mu}}$. Since $\gamma_{0}$ increases with power and $\hat{\mu}$ decreases with increasing edge temperature, it is not possible to ascertain the frequency scaling with power without a detailed transport model. The amplitude of the oscillations is such that at most the fluctuation level goes back to L-mode level. Figure 4 shows the comparison between the numerical results for $\omega$ and the analytical expression (9) as a function of $\hat{\mu}$. From the numerical solution of Eqs. (7) and (8), it is obtained that the number of cycles depends on the rate of increase of power. This type of behavior is similar to the dithering observed at the $\mathrm{L}$ to $\mathrm{H}$ 
transition [10].

\section{CONCLUSIONS}

In this paper, we have investigated a self-consisted model of the $\mathrm{L}$ to $\mathrm{H}$ transition by using primitive equations for the resistive interchange turbulence coupled with averaged shear flow evolution.

For low fluctuation levels, there are second order phase transitions between L-mode and H-mode type solutions. For large fluctuations, there is no flow generation. These results are consistent with the structure of the Reynolds stress.

A dithering behavior of the transition appears for very low viscosity (or high power). This behavior is due to the nonlinear modification of the shearing term in the fluctuation equations.

\section{ACKNOWLEDGMENTS}

This research was sponsored in part by Dirección General de Investigación Científica y Técnica of Spain (PB93-0231-C02-01); and in part by the Office of Fusion Energy, U.S. Department of Energy, under contract DE-AC05-84OR21400 with Martin Marietta Energy Systems, Inc, and through grant No. DE-FG03-88ER-53275. One of us (L.G.) wants to acknowledge Asociación EURATOM-CIEMAT and Comunidad de Madrid for partial support during the completion of this research.

\section{REFERENCES}

[1] DIAMOND, P.H., LIANG, Y.-M., CARRERAS, B.A., TERRY, P.W., Phys. Rev. Lett. 72 (1994) 2565.

[2] WAGNER, F., et al., Phys. Rev. Lett. 49 (1982) 1408.

[3] CHARLTON, L.A., CARRERAS, B.A., Phys. Plasmas (in press).

[4] CARRERAS, B.A., LYNCH, V.E., GARCIA, L., DIAMOND, P.H., Phys. Fluids B 5 (1993) 1491.

[5] CARRERAS, B.A., LYNCH, V.E., GARCIA, L., Phys. Fluids B 5 (1993) 1795.

[6] BIGLARI, H., DIAMOND, P.H., TERRY, P.W., Phys. Fluids B 2 (1990) 1.

[7] GALANTI, B., SULEN, P.-L., Phys. Fluids A 3 (1991) 1778.

[8] CARRERAS, B.A., GARCIA, L., DIAMOND, P.H., Phys. Fluids 30 (1987) 1388.

[9] CARRERAS, B.A., NEWMAN, D., DIAMOND, P.H., LIANG, Y.-M., Phys. Plasmas (in press).

[10] ZOHM, H., Phys. Rev. Lett. 72 (1994) 222. 


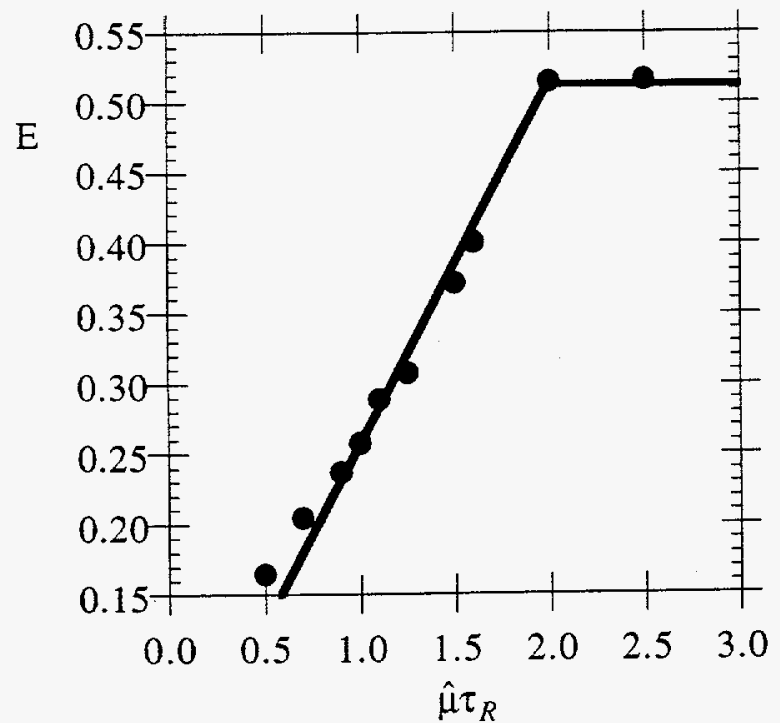

Fig. 1. Level of fluctuations $E$ at saturation versus $\hat{\mu}$ for $\beta_{0} / 2 \varepsilon^{2}=0.0075$.

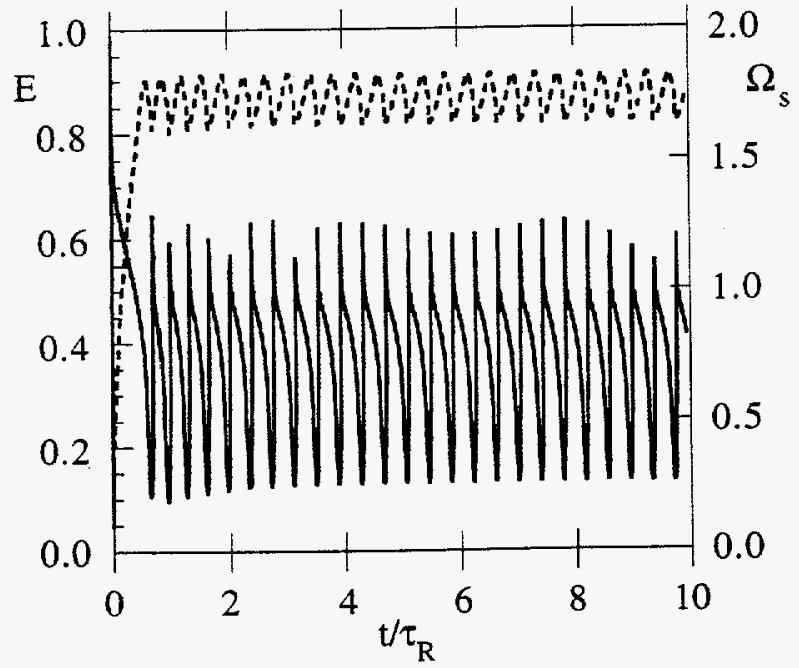

Fig. 3. Time evolution of the level of fluctuations $E$ (solid line) and the normalized shearing rate (broken line) for $\beta_{0} / 2 \varepsilon^{2}=0.01$ and $\hat{\mu}=\tau_{R}^{-1}$.

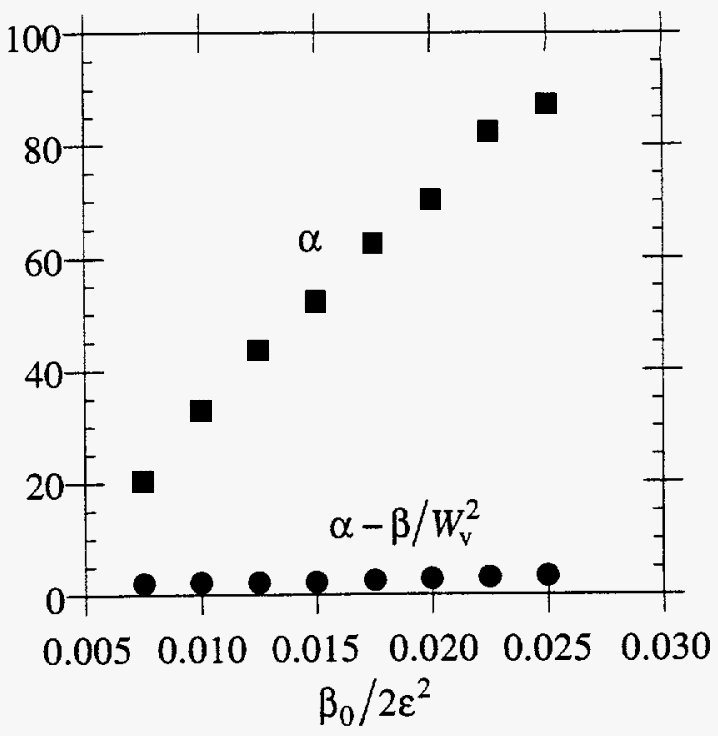

Fig. 2. The $\alpha$ and $\beta$-terms of the Reynolds stress versus $\beta_{0} / 2 \varepsilon^{2}$ for the equilibrium parameters of Ref. 4.

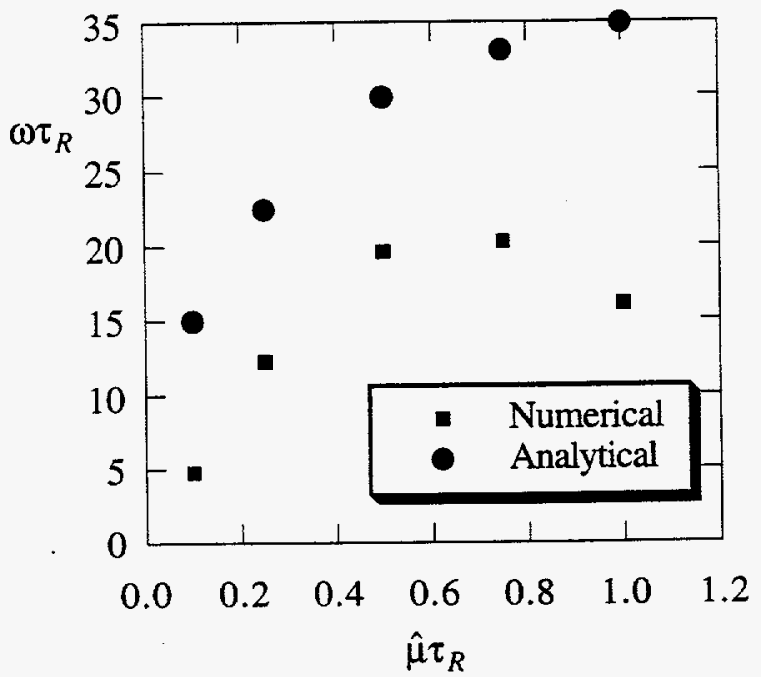

Fig. 4. Frequency of the oscillations versus $\hat{\mu}$ obtained from the numerical calculations and the analytical expression (9). 